\title{
Ultrasound Neurography in the Evaluation of Sciatic Nerve Injuries
}

\author{
AyHan BILGidi, MD ${ }^{1)}$, Cengiz ÇOKLuK, MD²), Keramettin Aydin, MD²) \\ 1) Department of Physical Therapy and Rehabilitation, Medical Faculy, Ondokuzmayis University \\ 2) Department of Physical Therapy and Neurosurgery, Medical Faculy, Ondokuzmayis University: \\ Samsun / Turkey. \\ TEL: +90 36231219 19/3086, FAX: +90 36245760 49,E-mail: cengizcokluk@yahoo.com
}

\begin{abstract}
Purpose] The aim of this clinical study was to investigate the benefits and the basic principles of ultrasonographic examination in the evaluation of sciatic nerve injuries. [Subjects and Methods] Patients with sciatic nerve injury were evaluated using a real-time utrasonographic examination. The capabilty of ultrasonography in terms of determination of the type and the localization of injury, the position of the proximal and distal nerve segments, the presence or absence of a neuroma, and perilesional scar tissue were evaluated in all cases. [Results] Ten cases with sciatic nerve injury were evaluated with real time sonography. Perilesional scar tissue formation was found in $4(40 \%)$ cases. Two $(20 \%)$ cases had stump neuroma diagnosed by sonographic examination. The capability of ultrasonographic examination was satisfactory for all evaluation parameters. [Conclusion] Ultrasonographic examination of sciatic nerve lesions may be used for the description of the degree of injury, determination of complete or incomplete nerve sectioning, the presence of hematoma and foreign body, the continuity of nerve, determination of nerve stumps, formation of perilesional scar tissue, and the presence of neuroma. Key words: Peripheral nerve injury, Ultrasonography, Sciatic nerve
\end{abstract}

(This article was submitted Feb. 28, 2011, and was accepted Apr. 6, 2011)

\section{INTRODUCTION}

The sciatic nerve is one of the thickest peripheral nerves in the body. This nerve leaves the pelvic cavity via the greater sciatic notch. The other anatomical structures following this nerve at this location are the pudendal nerve, the posterior femoral cutaneous nerve, the inferior gluteal vessels and the internal pudendal vessels. The sciatic nerve ends at the popliteal fossa after dividing into two terminal branches; the tibial and fibular nerves. The term "ultrasonographic neurography of the sciatic nerve" is used to describe the imaging of the sciatic nerve at the locations of the greater sciatic notch and the popliteal fossa where the nerve divides into its two terminal branches. The sciatic nerve may be injured by different causes. Trauma is one of them. Other causes resulting in function loss are tumors and entrapment neuropathies. Penetrating, oppressive, sharp and hard objects such as knives, glass and missiles are frequent causes of sciatic nerve injuries. Also, intramuscular injection for treatment may injure the nerve if they are injected close to or within the sciatic nerve.

History, physical and neurological examination, and electro-diagnostic tests are generally used for diagnosing of pathology 1-3). Electro-diagnostic tests are the gold standard in diagnosing a nerve lesion. However, especially in acute stage of injury, these tests may not yield reliable information. Also, electro-diagnostic tests may not be able to give any information about localization and description of injury, visualization of nerve stumps, diagnosis of a neuroma, evaluation of perilesional tissue and foreign bodies in the acute stage and chronic period. Because of this, a physician needs a reliable, cheap, practical, and readily available diagnostic method in clinical practice. Ultrasonographic evaluation can be used for the evaluation of the sciatic nerve; for diagnosis and evaluation of the injury site. Some clinical studies previously published in the literature emphasize ultrasound can be used for this purpose. The data obtained from patients with sciatic nerve injury and our clinical experience of ultrasonographic examination of injured sciatic nerves are presented here.

\section{SUBJECTS AND METHODS}

The study population was 10 patients ( 3 female and 7 male) with sciatic nerve injury who we treated between 2005 and 2008.

Tosbee ultrasound (Toshiba inc. Tokyo) with a 5-7, 5 $\mathrm{MHz}$ linear probe was used for the sonographic evaluation. Patients were positioned in the prone position. Ultrasound gel was applied to the probe surface and skin to enhance the visualization of peripheral nerves and musculoskeletal structures of the lower extremity. Before starting the ultrasonographic examination we grossly determined and localized the topographical injury site by gross evaluation of skin indents. We also took into account the result of electro-diagnostic studies and anatomical landmarks. Bone, 


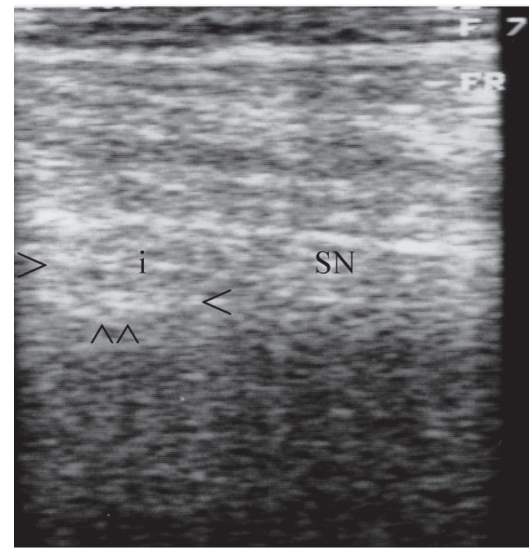

A

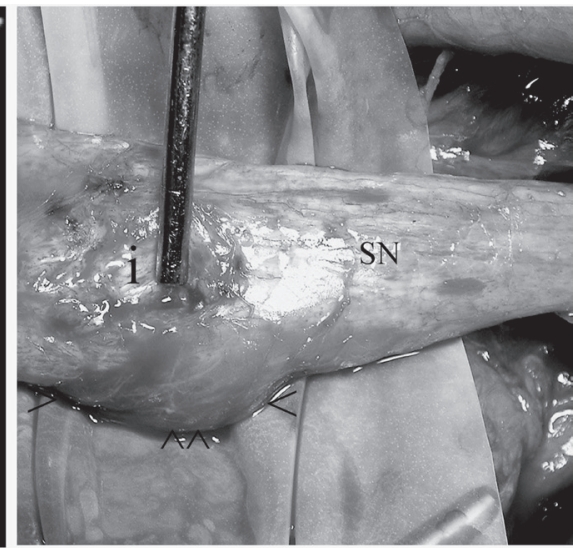

B

Fig. 1A. Sonographic examination revealed the injury site, neuroma formation and sciatic nerve (arrowheads show neuroma formation; i: injury site; SN: sciatic nerve) B. Intraoperative photograph shows injury site, neuroma formation and sciatic nerve (arrowheads show neuroma formation; i: injury site; SN: sciatic nerve).

muscles, tendons, vascular structures, and peripheral nerves were identified and distinguished. The continuity, architecture, shape, calibration and integrity of the injured sciatic nerve segment and peripheral tissues were examined in perpendicular and transverse planes. Still images were displayed on the monitor screen and printed on paper for archives and further examination. Sonographic findings of the patients who underwent surgery were compared with gross surgical observations.

We examined the capability of ultrasonographic examination in terms of visualization and identification of the sciatic nerve, localization of injury, description of the type of injury, determination of nerve stumps and foreign bodies, diagnosis of a neuroma, and evaluation of perilesional scar tissue. Ultrasonographic evaluations of these seven parameters were graded as poor, good, or excellent according to the examiner's description. Poor was scored as 1, good as 2, excellent as 3. Data are expressed as the mean standard error of the mean score. Differences in mean score between poor and excellent/good results were analyzed using Student's t-test and values of $\mathrm{p}<0,001$ were considered significant.

\section{RESULTS}

The 10 cases were 3 females and 7 males, aged between 15 and 55 years old. Their mean age was $40.2 \pm 19.54$ years and the female to male ratio was $3 / 7$.

In all cases the nerve was injured by penetrating objects or non-penetrating insults. Four cases $(40 \%)$ were injured by knife or metallic objects, one (10\%) by civilian gun-shot, one $(10 \%)$ by squeezing, and the remaining $4(40 \%)$ by injection of an injectable drug near or into the sciatic nerve.

Three cases $(30 \%)$ were acute injury (first 72 hours from injury) on admission; and the remaining 7 (70\%) were chronic (after 72 hours from injury) cases.

Perilesional scar tissue formation was found in 4 cases $(40 \%)$ and one case $(10 \%)$ cases had a neuroma diagnosed by sonographic examination. Ultrasound imaging (Fig. 1A) shows neuroma formation and the injured area. An intraoperative image is shown in Fig. 1B. Two cases (20\%) had foreign bodies at the injury site. The capability of ultrasonographic examination was satisfactory in all cases in terms of visualization and identification of the sciatic nerve, localization of the injury, description of the type of injury, determination of foreign bodies, diagnosis of stump neuroma, and determination of perilesional excessive scar tissue formation. Fig. 2 shows the injured site and swelling of the sciatic nerve and perilesional scar tissue formation around the nerve.

Visualization and identification of the sciatic nerve was found the good/excellent in $70 \%$ of the cases, and poor in $30 \%$ of the cases. Localization of the injury site by sonographic examination was found to be good/excellent in $70 \%$ of the cases and poor in $30 \%$ of the cases.

\section{DISCUSSION}

Do we need imaging modalities for the sciatic nerve? The answer to this question is clear. Gross morphological imaging of the sciatic nerve is necessary for better understanding of the pathology of affected nerve fibers. In injury cases the neuroradiological imaging modalities are specifically necessary for gaining advanced information about the condition of the sciatic nerve. Functionally, the sciatic nerve contains both motor and sensorial functions. Electrophysiological studies are important in the evaluation of the functional status of the nerve. At the same time a neurological examination may reveal the functional status of the sciatic nerve.

Electro-diagnostic studies cannot give any objective information about the morphological status of the nerve such as continuity, architecture, shape, diameter, or integrity of the sciatic nerve. An imaging method for the morphological examination of the sciatic nerve is needed. This imaging modality is needed for the acute and chronic stages 


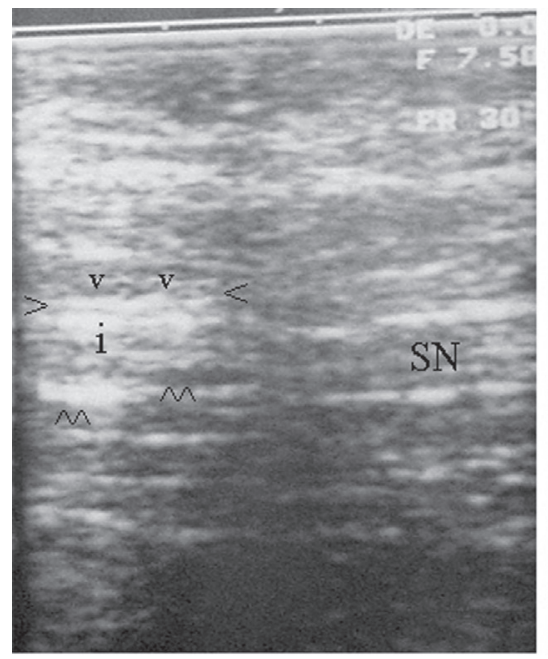

A

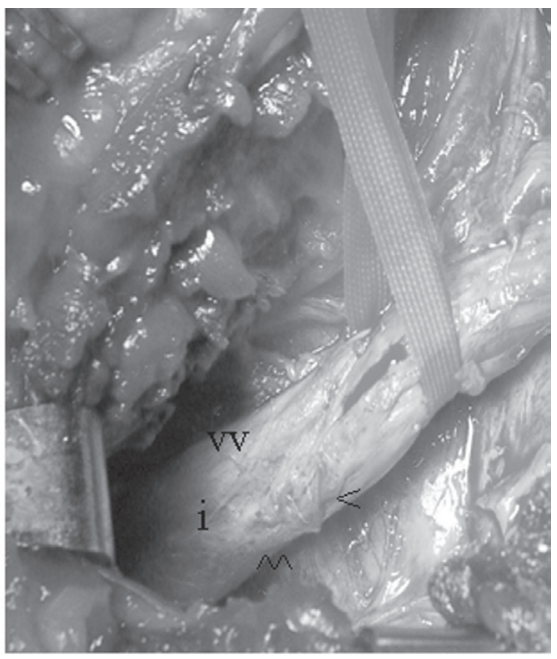

B

Fig. 2A. Sonographic examination revealed the injury site (arrowheads show swelling and fibrotic tissue formation around injured area; i: injury site; SN: sciatic nerve) B. Intraoperative photograph shows swelling, perilesional fibrous tissue formation and sciatic nerve (arrowheads show swelling and fibrous tissue formation around the injured area; i: injury site; SN: sciatic nerve).

of injury and it should be helpful for demonstrating the injury site and status of the nerve.

In the acute stage, the degree of injury, determination of complete or incomplete nerve sectioning, the presence of hematoma and foreign body can be provided by sonographic examination. In the chronic stage, the continuity of the nerve, determination of nerve stumps, perilesional scar tissue, and the presence of neuroma can be demonstrated by ultrasonography. The nerve can be sutured primarily after obtaining data from ultrasonographic neurography. The question "is the integrity of the sciatic nerve is conserved?" need to be answered. If the anatomical integrity of the sciatic nerve is injured it can be primarily repaired.

We hypothesized that ultrasonographic examination of the lower extremity may offer some reliable information about the patho-morphological status of injured sciatic nerves including detection of hematoma and foreign bodies. Ultrasonography is a real-time, mobile, and radiation free imaging technique. It may be able to show continuity, architecture, shape, diameter, and integrity of a nerve. Some previous studies have evaluated peripheral nerves by ultrasound4-7). In these studies, the authors described the normal ultrasonographic appearances of peripheral nerves4-7). Computerized tomography (CT) and magnetic resonance imaging (MRI) can also be used for neuroradiological imaging of nervous and musculoskeletal tissues. MRI has higher capability than CT for this purpose; but it does not provide real time images, and it is necessary to reconstruct images to determinate and distinguish of nerves. The results of our present study demonstrate that ultrasonography can be helpful for imaging of the sciatic nerve in the acute and chronic stages of injury.

This clinical study aimed to evaluate the ability of ultrasonography in the examination of sciatic nerve injuries. Our study reveals that ultrasonography in sciatic nerve injuries is a cheap, simple and readily available diagnostic tool for clinical practice. Future detailed studies are necessary to determine the grading of lesions and the grouping of injury types for use in ultrasonographic evaluation.

\section{REFERENCES}

1) Cokluk C, Aydin K, Senel A: Presurgical ultrasound assisted neuroexamination in the surgical repair of peripheral nerve injury. Minim Invasive Neurosurg 2004, 47: 169-172.

2) Gentili F, Hudson AR, Midha R: Peripheral nerve injuries: Types, causes, and grading. In: Neurosurgery (Vol 3). New York: McGraw-Hill, 1996: pp 3105-3114.

3) Kline DG, Hudson AR: Acute injuries of peripheral nerves. In: Neurological surgery (Vol 4). Philadelphia: W. B. Saunders Company, 1990: pp 2423-2510.

4) Cokluk C, Aydin K: Ultrasound examination in the surgical treatment for upper extremity peripheral nerve injuries: part I. Turk Neurosurg. 2007, 17: $277-282$.

5) Aydin K, Cokluk C, Pişkin A, et al.: Ultrasonographically checking the sectioning of the transverse carpal ligament during carpal tunnel surgery with limited uni skin incisions. Turk Neurosurg. 2007,17: 219-223.

6) Peer S, Bodner G, Meirer R, et al.: Examination of postoperative peripheral nerve lesions with high-resolution sonography. AJR 2001, 177: 415-419.

7) Cokluk C, Aydin K: Ultrasound examination in the surgical treatment of lower extremity peripheral nerve injuries: part II. Turk Neurosurg. 2007, 17: 197-201. 